\title{
Chiropractic services in the active duty military setting: a scoping review
}

Silvano Mior ${ }^{1,2^{*}}$ (D), Deborah Sutton ${ }^{1}$, Daphne To $^{3}$, Carolina Cancelliere ${ }^{1,4}$, Simon French ${ }^{5}$, Anne Taylor-Vaisey ${ }^{1}$ and Pierre Côté ${ }^{1,6}$

\begin{abstract}
Background: Musculoskeletal injuries are one of the most prevalent battle and non-battle related injuries in the active duty military. In some countries, chiropractic services are accessed to manage such injuries within and outside military healthcare systems; however, there is no recent description of such access nor outcomes. This scoping review aimed to synthesize published literature exploring the nature, models, and outcomes of chiropractic services provided to active duty military globally.

Method: We employed scoping review methodology. Systematic searches of relevant databases, including military collections and hand searches were conducted from inception to October 22, 2018. We included peer-reviewed English literature with qualitative and quantitative designs, describing chiropractic practice and services delivered to active duty military worldwide. Paired reviewers independently reviewed all citations and articles using a two-phase screening process. Data from relevant articles were extracted into evidence tables and sorted by study type. Results were descriptively analyzed.
\end{abstract}

Results: We screened 497 articles and 20 met inclusion criteria. Chiropractic services were commonly provided onbase only in the US. Services were accessed by physician referral and commonly after initiation or non-response to other care. Use of scope of practice was determined by the system/facility, varying from intervention specific to comprehensive services. Back pain with and without radiculopathy accounted for most complaints. Treatment outcomes were reported primarily by case reports. However, two recent randomized trials reported improved pain, disability, and satisfaction when adding chiropractic care to usual medical care compared to usual medical care alone in management of low back pain. Specific reaction time measures in special operation forces military did not improve after chiropractic care compared to wait-list control.

Conclusions: Our scoping review found the majority of published articles described chiropractic services in the active duty military in the US setting. Recent RCTs suggest a benefit of including chiropractic care to usual medical care in managing back pain in active duty military. Yet despite reported benefits in Australia, Canada, and the US, there is a need for further qualitative, descriptive, and clinical trial data worldwide to inform the role of chiropractic services in active duty military.

Keywords: Military personnel, Active duty, Chiropractic, Military medicine

\footnotetext{
* Correspondence: SMior@cmcc.ca

'UOIT-CMCC Centre for Disability Prevention and Rehabilitation, University of Ontario Institute of Technology (UOIT) and Canadian Memorial Chiropractic College (CMCC), 6100 Leslie Street, Toronto, Ontario M2H 3J1, Canada

${ }^{2}$ Department of Research and Innovation, Canadian Memorial Chiropractic

College, 6100 Leslie Street, Toronto, Ontario M2H 3J1, Canada

Full list of author information is available at the end of the article
}

(c) The Author(s). 2019 Open Access This article is distributed under the terms of the Creative Commons Attribution 4.0 International License (http://creativecommons.org/licenses/by/4.0/), which permits unrestricted use, distribution, and reproduction in any medium, provided you give appropriate credit to the original author(s) and the source, provide a link to the Creative Commons license, and indicate if changes were made. The Creative Commons Public Domain Dedication waiver (http://creativecommons.org/publicdomain/zero/1.0/) applies to the data made available in this article, unless otherwise stated. 


\section{Background}

Musculoskeletal injuries significantly affect the health and operational readiness of active military personnel. They are one of the most prevalent battle and non-battle related injuries in theatre [1, 2]. Analysis of United States (US) Navy Physical Evaluation Board data between February 2005 and February 2006 indicated that musculoskeletal diagnoses were frequent (43\%), with back pain (29\%) being the most common musculoskeletal diagnosis [3]. Musculoskeletal injuries are also one of the most common reasons for Canadian Armed Forces (CAF) personnel not being deployed [4], and were responsible for $42 \%$ of all medical releases in 2013 [5]. In addition, neck pain is an important aeromedical problem. Fiftyone percent $(51.7 \%)$ of Swedish Air Force aviators reported experiencing neck pain [6], while $53.3 \%$ of rotary-wing crew and $69 \%$ of fast-jet crew reported neck pain in the Royal Air Force [7].

In the active military setting, musculoskeletal conditions are associated with lost productivity due to sick parade attendance, lost duty days $[1,8,9]$, and impact the ability to deploy [5]. The probability of returning to full duties decreases with time spent away from duties [10], and the potential long term sequelae include limited duty assignment or early termination of service [8]. In addition to related costs for treatment interventions, medical discharge increases resource expenditures resulting from the recruitment and training of replacement recruits [2].

Evidence-based interventions for musculoskeletal injuries include a focus on active versus passive treatment, structured education, exercise, and manual and cognitive behavioural therapies [11]. Musculoskeletal programs of care in the military setting are frequently delivered in a multidisciplinary healthcare environment. Access to these programs of care by military personnel most often occurs through a traditional gatekeeper physician referral [12], or through a non-traditional gatekeeper such as a physical therapist [13]; personnel are referred to other musculoskeletal healthcare providers, or to team assessment and management $[14,15]$.

Available reviews have compared or described chiropractic services within both military and veteran healthcare systems in combination [16-18]. A 2009 review described chiropractic services in military and veteran healthcare systems in the US and Canada, but concluded that there was a need to evaluate the processes, policies, practices, and effectiveness of chiropractic services in these settings [17]. However, no recent knowledge syntheses have summarized the integration of chiropractic services in global military healthcare systems solely within an active duty military population. In particular, to our knowledge there is no current review of the literature describing chiropractic services and its utilization, scope of practice, and policies in the active duty military worldwide. Such a review can assist in informing the role of chiropractic services in this population.

Therefore, the objective of this scoping review was to document the current global state of knowledge related to chiropractic services in the active duty military setting with respect to: 1) access of chiropractic services; 2) chiropractic scope of practice, e.g. procedures, processes, and actions; 3) service model and location; and 4) type of condition treated, duration, and outcomes of treatment provided to active duty military members.

\section{Methods}

We employed scoping review methodology to collect and organize relevant information to synthesize the available evidence addressing our broad research question [19]. We applied the scoping review framework of Arksey and O'Malley [19] and successive recommendations [20-22] for conducting and reporting scoping reviews. Consistent with this framework, we did not critically appraise the methodology of reviewed articles [19-21]. This review is reported against the PRISMA extension for scoping reviews (PRISMA-ScR) [22].

\section{Stage 1: identifying the research question}

Our scoping review was guided by the following broad research question: What is published in the peerreviewed literature regarding the access, scope of practice, service models, conditions treated and outcomes related to chiropractic care for active duty military members?

\section{Stage 2: identifying relevant articles}

Our search strategy was developed in consultation with a health sciences librarian, and a second librarian reviewed the search for completeness using the Peer Review of Electronic Search Strategies (PRESS) Checklist [23, 24]. The search strategy was first developed in MEDLINE (Ovid ${ }^{\circ}$ ) and subsequently adapted to the other databases. The search terms included subject headings specific to each database (e.g., MeSH in MEDLINE) [25] and free text words relevant to utilization of chiropractors and chiropractic services were combined with terms relevant to the army and active duty military (see Additional file 1 for full MEDLINE search strategy).

We searched MEDLINE, Ovid MEDLINE In-Process and Other Non-Indexed Citations, PsycINFO, Cochrane Central Register of Controlled Trials, and Embase, through Ovid Technologies, Inc.; CINAHL Plus through EBSCOhost from inception to September 15, 2018; and the Military \& Government Collection through EBSCOhost; and the Military Database through ProQuest from inception to October 22, 2018. The reference lists of relevant articles were hand searched for additional articles not identified from the electronic database search. 
We used the PRISMA-ScR [22] flow chart to track the number of articles at each stage of the review. The results from the database searches were combined and imported to EndNote X6 [26]. We did not register this review prior to undertaking it.

\section{Stage 3: article selection}

\section{Inclusion and exclusion criteria}

Eligible studies met the following criteria: 1) published in the peer-reviewed literature; 2) written in the English language; 3) were any primary qualitative or quantitative designs, including qualitative studies, randomized controlled trials, quasi-randomized trials, cohort, crosssectional, case report and case series designs; 4) described chiropractic services; and 5) the study population included active duty military personnel, the National Guard, or reservists. Study exclusion criteria included: narrative and systematic reviews, letters, editorials, commentaries, unpublished manuscripts, dissertations, government reports, books and book chapters, conference proceedings, meeting abstracts, lectures and addresses, consensus development statements, informal communication, e.g. blogs, podcasts, email, cadaveric or animal studies, and nonactive military members, e.g. veterans.

\section{Screening and agreement}

Eligible articles were selected through a two-phase screening process. In Phase 1, two of the authors (DS, DT) independently screened titles and abstracts to determine eligibility. Articles were classified as relevant, possibly relevant, or irrelevant. In Phase 2, the same reviewers independently reviewed full text manuscripts of relevant and possibly relevant articles to make a final determination of eligibility. Reviewers met to solve disagreements and reach consensus in both phases. We involved a third independent reviewer (SM) if consensus could not be reached. We contacted authors when additional information was needed to confirm article relevance.

\section{Stage 4: data charting}

We extracted the following data from the relevant articles (when available): 1) study description (study design, country of origin, service model and branch, and study population); 2) type of condition and duration; 3 ) chiropractic services provided; and 4) study findings (e.g. utilization of chiropractic services, patient outcomes, satisfaction). One review author (DS) extracted the data which were independently checked by a second review author (SM) to minimize error.

Stage 5: collating, summarizing, and reporting the results We employed a 'descriptive-analytical' method within the narrative tradition to summarize the data and include the following [27]:
1. Descriptive numerical analysis: The nature and distribution of the articles were examined with respect to the total number of articles, year of publication, country where studies were conducted, study population, and study design.

2. Narrative summary of included study findings: We classified the studies according to our review objectives: 1) access to chiropractic services; 2) chiropractic scope of practice, e.g. procedures, processes, and actions; 3 ) service model and location of delivery; and 4) type of condition treated, duration and outcomes of treatment provided for active duty military members globally. Where relevant and where possible, we extracted the $95 \%$ confidence intervals around any point estimates provided.

3. Implication of results: We reported the findings according to our objective of describing the published literature on utilization, scope of practice, and policies related to chiropractic services for active duty military members globally.

\section{Results}

Our search yielded 674 citations. We removed 154 duplicates and screened 520 articles (Fig. 1). During Phase I screening, we excluded 461 articles, and a further 39 articles following Phase II screening. We contacted two authors for clarification, one regarding military status of participants and one to clarify treatment specifics of chiropractic care provided. However, neither author responded; these two articles were excluded. Twenty articles were included in this review [12, 16, 28-45].

\section{Descriptive numerical analysis}

Table 1 summarizes the key findings from the relevant articles. Research on the utilization, scope of practice, and policies related to chiropractic services for active duty military members globally is a small and relatively recent body of literature, with the earliest included study published in 2006. The studies were most commonly conducted in the United States $(n=17)$ [16, 28-32, 34-43, 45], with one conducted in Australia $(n=1)$ [33], and two in Canada $(n=2)[12,44]$. Most studies were cross-sectional $(n=9)[12,28,31-37]$, six were case reports [38-43], three were randomized controlled trials [29, 30, 45], and two were qualitative designs $[16,44]$.

\section{Location and access to chiropractic services}

Six case reports [38-43], three randomized controlled trials [29, 30, 45], and one cross-sectional study [12] described chiropractic services provided to active duty military personnel worldwide. In North America, chiropractic services were reported as initiated through referral from a primary care provider (gatekeeper) following 


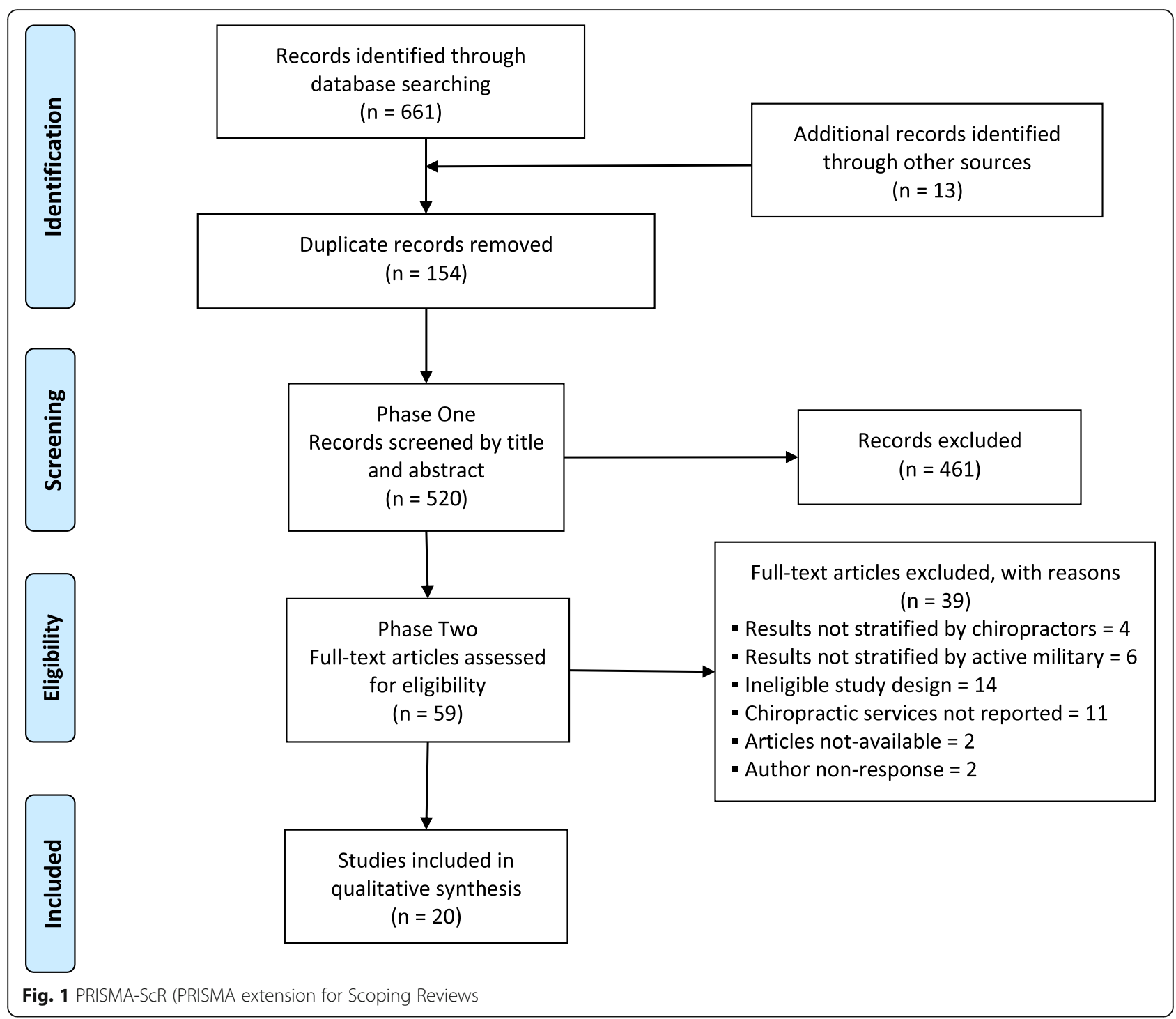

initial assessment, except in the randomized controlled studies where access was predetermined by study design $[29,30,45]$.

As reported in the included articles, patients typically accessed chiropractic services through a gatekeeper, and were seen primarily for musculoskeletal conditions $[16,44]$. The referral for chiropractic services may occur after the initial assessment, but most often occurred after initiation and non-response from other interventions [16, 41, 44]. Such other interventions included prescribed medication, diagnostic imaging, e.g. radiographs, magnetic resonance imaging, physical therapy, and referral to other healthcare services, e.g. pain clinic, specialist consultation. Direct access by active duty military personnel to chiropractic care was not reported in any included study.

Dunn et al. [16] in their qualitative study noted that chiropractic care was initiated into the US Department of Defense (DoD) in response to legislative action (1993-2009), and service has grown to 49 Military Health System commands. A survey to identify complementary and alternative medicine use at fourteen Military Treatment Facilities reported $92 \%$ of these facilities offered chiropractic services in 2005 compared to $85 \%$ in 2009 [34]. Additionally, the Military Health System Complementary and Alternative Medicine survey identified 55\% of US Military Treatment Facilities offered chiropractic services in 2013 [31]. However, in 2005, 54\% of active duty personnel resided in areas served by chiropractic clinics, with the remainder not served because of living overseas $(14 \%)$, in remote areas $(5 \%)$, or on bases with no chiropractic clinics (28\%) [28].

In one Canadian study [12], chiropractic care was provided on-base in one location; however, this is no longer the case. Currently in Canada, chiropractic care is an eligible health benefit to CAF personnel and is accessed off-base, outside the military health system [44]. 
Table 1 Summary of location, population, condition of interest and key findings reported in included studies $(n=20)$

\begin{tabular}{|c|c|c|c|c|c|}
\hline $\begin{array}{l}\text { First } \\
\text { Author, } \\
\text { Year }\end{array}$ & $\begin{array}{l}\text { Country, Service } \\
\text { Branch, Service } \\
\text { Location, }\end{array}$ & $\begin{array}{l}\text { Population, } \\
\text { Number, } \\
\text { Groups }\end{array}$ & $\begin{array}{l}\text { Condition and } \\
\text { Duration }\end{array}$ & Treatment & $\begin{array}{l}\text { Key Findings: visit-specific } \\
\text { information, outcomes, } \\
\text { adverse events }\end{array}$ \\
\hline & $\begin{array}{l}\text { Randomized } \\
\text { Control Trials }\end{array}$ & & & & \\
\hline \multirow[t]{13}{*}{$\begin{array}{l}\text { DeVocht } \\
2019[45]\end{array}$} & \multirow{13}{*}{$\begin{array}{l}\text { United States } \\
\text { Blanchfield Army } \\
\text { Community } \\
\text { Hospital, KY } \\
\text { SOF }\end{array}$} & $\begin{array}{l}\text { Personnel On- } \\
\text { base }\end{array}$ & \multirow{13}{*}{$\begin{array}{l}\text { Little or no } \\
\text { body pain } \\
\text { (avg pain intensity } \\
<4 \text { on } 10 \text { scale) }\end{array}$} & \multirow{2}{*}{$\begin{array}{l}\text { CMT: HVLA SMT to } \\
\text { cervical, thoracic, } \\
\text { lumbopelvic areas, } \\
\text { as indicated }\end{array}$} & $\begin{array}{l}\text { Mean age } 33 \pm 5.6 \text { years; } \\
\text { male } 100 \%\end{array}$ \\
\hline & & \multirow{12}{*}{$\begin{array}{l}\text { CMT } 4 \text { visits over } \\
2 \text { weeks }(n=60) \\
\text { vs Wait-list Control } \\
(n=60)\end{array}$} & & & $\begin{array}{l}\text { Pain intensity: median } \\
\text { (range): } 2.0(0-3.0)\end{array}$ \\
\hline & & & & $\begin{array}{l}\text { Wait-list Control: } \\
\text { no treatment }\end{array}$ & $\begin{array}{l}\text { Primary Outcome: Mean } \\
\text { change }(95 \% \mathrm{CI}) \text { between } \\
\text { CMT and wait-list control } \\
\text { at } 2 \text { weeks not statistically } \\
\text { significant: }\end{array}$ \\
\hline & & & & & $\begin{array}{l}\text { Hand simple reaction } \\
\text { time: }-3.49 \text { (-24.75 to } 18.77)\end{array}$ \\
\hline & & & & & $\begin{array}{l}\text { Foot simple reaction time: } \\
0.97(-18.04 \text { to } 19.98)\end{array}$ \\
\hline & & & & & $\begin{array}{l}\text { Choice reaction time: } 3.49 \\
(-14.40 \text { to } 21.39)\end{array}$ \\
\hline & & & & & $\begin{array}{l}\text { Fitt's Law test response } \\
\text { time: } 0.99(-0.37 \text { to } 2.35)\end{array}$ \\
\hline & & & & & $\begin{array}{l}\text { t-wall response time: }-0.41 \\
(-1.24 \text { to } 0.41)\end{array}$ \\
\hline & & & & & Secondary Outcome: \\
\hline & & & & & $\begin{array}{l}\text { Mean change }(95 \% \mathrm{Cl}) \text { pre- } \\
\text { and post-reaction response } \\
\text { time at visit } 2 \text { and final visit } \\
\text { in favor of CMT for t-wall } \\
\text { response time only. }\end{array}$ \\
\hline & & & & & $\begin{array}{l}\text { Visit } 2 \text { t-wall response } \\
\text { time:-0.90 }(-1.71 \text { to }-0.09)\end{array}$ \\
\hline & & & & & $\begin{array}{l}\text { Final Visit t-wall response } \\
\text { time: }-0.75(-1.43 \text { to }-0.06)\end{array}$ \\
\hline & & & & & $\begin{array}{l}\text { Adverse events: } 0 \text { related } \\
\text { to trial procedures } 4 \text { related } \\
\text { to activities }\end{array}$ \\
\hline \multirow{13}{*}{$\begin{array}{l}\text { Goertz, } \\
2013[29]\end{array}$} & \multirow[t]{13}{*}{ United States } & \multirow{13}{*}{$\begin{array}{l}\text { On-base } \\
\text { personnel }\end{array}$} & \multirow{13}{*}{$\begin{array}{l}\text { Acute LBP } \\
(<4 \text { wks })\end{array}$} & \multirow{4}{*}{$\begin{array}{l}\text { CMT: including } \\
\text { HVLA SMT, } \\
\text { massage, exercises, } \\
\text { McKenzie exercises, } \\
\text { mobilization, advice- } \\
\text { ADL, postural, } \\
\text { ergonomic }\end{array}$} & Mean age 26 years; male $86 \%$ \\
\hline & & & & & $\begin{array}{l}\text { Mean duration of complaint } \\
9 \text { days }\end{array}$ \\
\hline & & & & & $\begin{array}{l}\text { Radicular signs in } 43 \% \text { of } \\
\text { participants }\end{array}$ \\
\hline & & & & & $\begin{array}{l}\text { Mean visits SMC 1.4; mean } \\
\text { SMC } 1+\text { CMT } 7\end{array}$ \\
\hline & & & & \multirow{4}{*}{$\begin{array}{l}\text { SMC: include usual care, } \\
\text { medications, physical } \\
\text { therapy, pain clinic }\end{array}$} & $\begin{array}{l}\text { Mean difference favouring } \\
\text { SMC + CMT at } 2 \text { weeks: }\end{array}$ \\
\hline & & & & & RMDQ $3.9(95 \% \mathrm{Cl} 1.8,6.1)$; \\
\hline & & & & & NRS $1.2(95 \% \mathrm{Cl} 0.2,2.3) ;$ \\
\hline & & & & & BPFS $-7.7(95 \% \mathrm{Cl}-12.9,-2.6$ \\
\hline & & & & \multirow[t]{5}{*}{$\begin{array}{l}2 \text { visits weekly } \\
\text { over } 4 \text { weeks }\end{array}$} & $\begin{array}{l}\text { Mean difference favouring } \\
\text { SMC + CMT at } 4 \text { weeks: }\end{array}$ \\
\hline & & & & & RMDQ $4.0(95 \% \mathrm{Cl} 1.3,6.7) ;$ \\
\hline & & & & & NRS $2.2(95 \% \mathrm{Cl} 1.2,3.1)$ \\
\hline & & & & & BPFS $-10(95 \% \mathrm{Cl}-14.6,-5.5)$ \\
\hline & & & & & SMC vs SMC + CMT satisfaction \\
\hline
\end{tabular}


Table 1 Summary of location, population, condition of interest and key findings reported in included studies ( $n=20)$ (Continued)

\begin{tabular}{|c|c|c|c|c|c|}
\hline $\begin{array}{l}\text { irst } \\
\text { luthor, } \\
\text { ear }\end{array}$ & $\begin{array}{l}\text { Country, Service } \\
\text { Branch, Service } \\
\text { Location, }\end{array}$ & $\begin{array}{l}\text { Population, } \\
\text { Number, } \\
\text { Groups }\end{array}$ & $\begin{array}{l}\text { Condition and } \\
\text { Duration }\end{array}$ & Treatment & $\begin{array}{l}\text { Key Findings: visit-specific } \\
\text { information, outcomes, } \\
\text { adverse events }\end{array}$ \\
\hline
\end{tabular}

with care (mean) at Week $2=4.5$ and 8.9 and at Week $4,5.4$ and 8.9 , respectively

Army

William Beaumont Army Medical Center

$\mathrm{SMC}+\mathrm{CMT}-2$

visits/wk. over

4 wks $(n=45)$

vs SMC $(n=46)$

Active duty

LBP (any duration) UMC with

Goertz,

2018 [30]

Army, Navy

Walter Reed National Military Medical Centre, Naval Medical Centre San Diego, Naval Hospital

Pensacola

service personnel

UMC with

chiropractic care

$(n=375) 12$ visits

over 6 wks vs

UMC $(n=375)$
Chiropractic Care: UMC plus up to 12 visits of chiropractic care including SMT, rehabilitative exercise, interferential current; ultrasound, cryotherapy, superficial heat, other manual therapies
UMC: include selfmanagement advice, pharmacologic pain management, physical therapy, pain clinic referral
Global Improvement (\% moderately better to completely gone): SMC 17\%; SMC + CMT 73\%

Participants had higher expectation of helpfulness with SMC + CMT

No follow-up assessments: SMC 35\%; SMC + CMT 15\%

No serious adverse events. Two mild, expected events reported in SMC + CMT group - 1 unrelated to intervention, 1 sharp pain in $L B$, referred for medication and resolved in $48 \mathrm{~h}$.

Mean age 30.9 (8.7) years; $23.3 \%$ female

Mean visits UMC with at least 1 visit to UMC clinician: Walter Reed 2.6 (2.3); San Diego 2.7(2.5); Pensacola 2.3 (2.3)

Mean visits UMC with chiropractic care with at least 1 visit to UMC clinician:

Walter Reed 2.6 (3.1); San Diego 3.5 (3.0); Pensacola $1.6(1.6)$

Mean visits to chiropractor with at least 1 chiropractic visit:

Walter Reed 4.7 (2.5);San Diego 2.3 (1.4); Pensacola $5.4(2.6)$

Mean duration (months)

UMC:

$<1144$ (38.4)

1-3 $40(10.7)$

$>3191(50.9)$

UMC with chiropractic care:

$<1143$ (38.1)

1-3 $39(10.4)$

$>3193(51.5)$

Primary Outcomes:

Differences observed at all 3 sites

Mean difference favoring UMC with chiropractic care at 6 weeks: 
Table 1 Summary of location, population, condition of interest and key findings reported in included studies ( $n=20)$ (Continued)

\begin{tabular}{|c|c|c|c|c|c|}
\hline $\begin{array}{l}\text { irst } \\
\text { luthor, } \\
\text { ear }\end{array}$ & $\begin{array}{l}\text { Country, Service } \\
\text { Branch, Service } \\
\text { Location, }\end{array}$ & $\begin{array}{l}\text { Population, } \\
\text { Number, } \\
\text { Groups }\end{array}$ & $\begin{array}{l}\text { Condition and } \\
\text { Duration }\end{array}$ & Treatment & $\begin{array}{l}\text { Key Findings: visit-specific } \\
\text { information, outcomes, } \\
\text { adverse events }\end{array}$ \\
\hline
\end{tabular}

NRS: $-1.1(95 \% \mathrm{Cl}-1.4$

to -0.7$)$

RMDQ: $-2.2(95 \% \mathrm{Cl}-3.1$

to -1.2 )

Mean difference favoring UMC

with chiropractic care at 12 weeks:

NRS (average): -0.9 (95\%Cl -1.2 to -0.5

RMDQ: -2.0 (95\% Cl -3.0 to -1.0 )

Secondary Outcomes:

Differences observed at all 3 sites

Mean difference favoring UMC with chiropractic care at 6 weeks:

NRS (worst): -1.2 (95\% Cl -1.6 to -0.8 )

Bothersomeness: -0.4 (95\% Cl -0.6 to -0.2 )

Mean difference favoring UMC

with chiropractic care at 12 weeks:

NRS (worst): -1.1 (95\% Cl -1.6 to -0.7 )

Bothersomeness: -0.4

(95\% Cl - 0.6 to - 0.2)

Significantly better global perceived improvement favoring UMC with chiropractic care at 6 weeks:Observed at all 3 sites OR 0.18 (95\% Cl 0.13 to 0.25 )

Significantly greater mean satisfaction with care favoring UMC with chiropractic care at 6 weeks:Observed at all 3 sites 2.5 ( $95 \% \mathrm{Cl} 1.6$ to 3.0 )

Significantly less pain medication

use favoring UMC with chiropractic care at: 6 weeks: OR $.73(95 \% \mathrm{Cl}$ 0.54 to 0.97 )

12 weeks: OR 0.76 (95\% Cl 0.58 to 1.00 )

No serious related adverse events. 62 events reported: UMC 
Table 1 Summary of location, population, condition of interest and key findings reported in included studies ( $n=20)$ (Continued)

First Country, Service Population, Condition and Treatment Key Findings: visit-specific

Author, Branch, Service Number, Duration

information, outcomes, adverse events

alone - 19 (3 medication

related, 4 epidural injections, 12 muscle/ joint stiffness

physiotherapy or self-care related.

UMC + chiropractic care - 43 (37 muscle/ joint stiffness related to chiropractic care and 1 related to physiotherapy care, 1 post epidural injection, 3 not treatment specified, 1 lower limb burning sensation 20 min post manipulation.

Cross-sectional Surveys

$\begin{array}{ll}\text { Boudreau, } & \text { Canada } \\ 2006 \text { [12] } & \text { Navy }\end{array}$

Navy

Archie McCallum

Hospital, CFB

Stadacona
On-base Patients, MSK

$n=102$ Physicians, complaints

$$
n=12
$$

Adjunct treatment: interferential current, acupuncture

Joint manipulation, soft tissue massage, stretching, exercise
Active duty

personnel

$n=30,664$
Patients - response rate 68\%; mean age $37 \mathrm{yr}$. (SD 8)

Presenting complaint: 97\% axial MSK complaints (52\% LBP), 3\% extremities; current episode: $41 \%$ acute, $56 \%$ chronic;Average visits/ patient: 5.7 (SD 4.1);

94.2\% were satisfied with chiropractic care

100\% agreed: office was easy to get to, attending DC treated them

with respect and concern 98.6\% agree DCs ability to answer questions; $98.5 \%$ high satisfaction with clinic hours of operation; $97.1 \%$ agreement that DC thought patients were important and was careful to check everything in the examination; $37.6 \%$ disagreed or unsure if DC office had appropriate equipment; $33.2 \%$ patients reported improvements took longer than expected; and $30.3 \%$ expected better results or were unsure if they should have expected better results

Physicians: 100\% perceived demand from patients for DC;80.6\% satisfied with DC services

Reasons for referral: axial MSK complaints, unresponsive to PT, patient request, PT waiting list too long, history of positive response to DC.

Response rate 51.8\%; 5.2\%

(0.46 SE) reported using chiropractic in preceding 12 months (male $4.9 \%$ (0.44 SE; female 6.9\% (0.96 SE) 
Table 1 Summary of location, population, condition of interest and key findings reported in included studies $(n=20)$ (Continued)

\begin{tabular}{ll}
\hline First & Country, Service \\
Author, & Branch, Service \\
Year & Location, \\
\hline & Army, Navy, Marine Corps, \\
& Air Force \\
& Stratified sample of 60 \\
& military installations by \\
& service and world region, \\
including afloat status & for Navy
\end{tabular}

$\begin{array}{ll}\text { Herman, } & \text { United States } \\ 2017 \text { [31] } & \text { Air Force, Army, National } \\ & \text { Capital Region Medical } \\ & \text { Directorate, Navy and } \\ & \text { Marine Corps }\end{array}$

Military treatment facilities

$\begin{array}{ll}\text { Population, } & \text { Condition and } \\ \text { Number, } & \text { Duration }\end{array}$

Key Findings: visit-specific information, outcomes, adverse events

ORs of using chiropractic: 30-39 years $2.26(95 \%$ Cl $1.08,4.74)$ and $40+$ years 3.42 (95\% Cl 1.36, 8.58) more likely than $<29$ years; Black/nonHispanic 0.35 (95\% Cl $0.19,0.66)$ less likely than White/non-Hispanic; 4 year college education 3.36 (95\% Cl 1.46, 7.72) more likely than high school education

Adjusted prevalence of chiropractic use (2005): $6.2 \%(0.62 \mathrm{SE})$ is less than NHIS (2002): $7.5 \%$ (0.19 SE) or NHIS (2007) 8.6\% (0.27 SE)

MTF, $n=142$

Response rate 94\% (133/142) 110 MTFs provided CAM services and 60 (55\%) of MTF offer chiropractic services; 5 reasons/ conditions for using chiropractic services $(n=49)$ : back pain 47 (42.7\%), chronic pain 44 (40.0\%), headache (excluding TBI related pain) $30(27.3 \%)$, acute pain (post trauma/injury, postop, preop 30 (27.3\%), general health/wellness/ prevention $12(10.9 \%)$; MHS (2013) number unique patients 55,843 ; average patient/visits 5.367; average procedures/ visit 1.05; MTF estimated number of chiropractic patient encounters 168,00/year Corps, Navy, Coast Guard, Active duty personnel, Reserve/National Guard

\section{Netto, 2011 Australia}

Air Force

$n=86,131$

Response rate Panel 1 71\%; Panel 2 25\%

$10.5 \%$ reported using chiropractic care in the preceding 12 months

\section{[33]}

RAAF Air Combat

Group $n=86$

Off base chiropractic care, which is accessed on a case-by-case basis usually after the failure of on-base services

Petri, United States

personnel
Response rate 95\% (82/86)

$78 \%$ of Royal Australian Air Force Fast Jet Aircrew experienced flight-related neck pain during or after a flight 55\% sought treatment for pain; 12\% sought chiropractic treatment for flight-related neck pain; 22\% reported chiropractic treatment most effective for flight-related neck pain

Response rate 2005 100\%, 2009 92.1\% MTFs: chiropractic 
Table 1 Summary of location, population, condition of interest and key findings reported in included studies ( $n=20)$ (Continued)

\begin{tabular}{|c|c|c|c|c|c|}
\hline $\begin{array}{l}\text { First } \\
\text { Author, } \\
\text { Year } \\
\end{array}$ & $\begin{array}{l}\text { Country, Service } \\
\text { Branch, Service } \\
\text { Location, }\end{array}$ & $\begin{array}{l}\text { Population, } \\
\text { Number, } \\
\text { Groups }\end{array}$ & $\begin{array}{l}\text { Condition and } \\
\text { Duration }\end{array}$ & Treatment & $\begin{array}{l}\text { Key Findings: visit-specific } \\
\text { information, outcomes, } \\
\text { adverse events }\end{array}$ \\
\hline & $\begin{array}{l}\text { Army }(n=8), \\
\text { Navy }(n=3), \\
\text { Air Force }(n=2), \\
\text { other }(n=1) \\
2005(n=14) \text { and } \\
2009(n=13)\end{array}$ & & & & $\begin{array}{l}\text { services available - } 2005 \\
(92 \%) \text { and } 2009(85 \%) ; \\
\text { providing individual } \\
\text { chiropractic services - } \\
2005(92 \%) \text { and } 2009 \\
(79 \%) ; \text { number of } \\
\text { chiropractors } 2005 \text { [15] } \\
\text { and } 2009 \text { [19] }\end{array}$ \\
\hline \multirow{3}{*}{$\begin{array}{l}\text { Ryan, } \\
2007 \text { [35] }\end{array}$} & United States & \multirow{3}{*}{$\begin{array}{l}\text { Active duty and } \\
\text { reserve personnel } \\
n=214,338\end{array}$} & & & \multirow{2}{*}{$\begin{array}{l}\text { Response rate } 36 \% \\
(77,047 / 214,338)\end{array}$} \\
\hline & \multirow{2}{*}{$\begin{array}{l}\text { Army, navy, Coast } \\
\text { Guard, Air Force, } \\
\text { Marines. }\end{array}$} & & & & \\
\hline & & & & & $\begin{array}{l}\text { Chiropractic care use: } \\
\text { Active duty } 8.0 \% \text {; Reserve/ } \\
\text { Guard } 14.8 \%\end{array}$ \\
\hline \multirow{4}{*}{$\begin{array}{l}\text { Smith, } \\
2008[36]\end{array}$} & United States & \multirow{2}{*}{$\begin{array}{l}\text { Population: } \\
\mathrm{n} \sim 550,000\end{array}$} & & & \multirow{4}{*}{$\begin{array}{l}\text { Response rate } 39 \% \\
\text { (1446/3683); Results } \\
\text { reported on } 1310 \text { of } \\
1372 \text { active duty; } 8.6 \% \\
\text { reported using } \\
\text { chiropractic care in the } \\
\text { preceding } 12 \text { months; } \\
\text { participants assisted by } \\
\text { practitioner with } \\
\text { chiropractic services } \\
\text { were at increased risk } \\
\text { of future hospitalization } \\
\text { compared to those self- } \\
\text { reporting such use (HR } \\
\text { 1.96; } 95 \% \text { Cl 1.01, 3.80) }\end{array}$} \\
\hline & \multirow{3}{*}{$\begin{array}{l}\text { Navy, Marine Corps, } \\
\text { Reserve Navy and } \\
\text { Marine Corps }\end{array}$} & & & & \\
\hline & & $\begin{array}{l}\text { In-patient and } \\
\text { out-patient }\end{array}$ & & & \\
\hline & & $\begin{array}{l}\text { Surveyed random } \\
\text { sample: } n=5000 \\
\text { but } 3683 \text { were } \\
\text { eligible }\end{array}$ & & & \\
\hline \multirow{4}{*}{$\begin{array}{l}\text { White, } \\
2011 \text { [37] }\end{array}$} & United States & \multirow{4}{*}{$\begin{array}{l}\text { In-patient and } \\
\text { out-patient } \\
\text { Surveyed random } \\
\text { sample active } \\
\text { duty personnel, } \\
n=44,287\end{array}$} & & & \multirow{4}{*}{$\begin{array}{l}29 \% \text { reported using at } \\
\text { least one practitioner } \\
\text { assisted CAM } 8.1 \% \\
\text { reported using } \\
\text { chiropractic care in the } \\
\text { preceding } 12 \text { months }\end{array}$} \\
\hline & $\begin{array}{l}\text { Army, Navy, Air } \\
\text { Force, Marine Corps, } \\
\text { Coast Guard }\end{array}$ & & & & \\
\hline & $\begin{array}{l}\text { Standard Inpatient Data } \\
\text { Record; DoD TRI-CARE } \\
\text { Management Activity's } \\
\text { Health Care Service Record, } \\
\text { Standard Ambulatory } \\
\text { Data Record }\end{array}$ & & & & \\
\hline & Case Report & & & & \\
\hline \multirow{5}{*}{$\begin{array}{l}\text { Green, } \\
2006 \text { [38] }\end{array}$} & United States & \multirow[t]{5}{*}{36 yo, male } & \multirow{5}{*}{$\begin{array}{l}\text { Acute non- } \\
\text { specific LBP }\end{array}$} & \multirow{5}{*}{$\begin{array}{l}\text { Interdisciplinary } \\
\text { treatment, with } \\
\text { chiropractic care } \\
\text { provided over } 16 \\
\text { visits in } 30 \text { weeks, } \\
\text { included HVLA SMT, } \\
\text { mobilization, active } \\
\text { myofascial release } \\
\text { therapy, exercise, } \\
\text { ischemic compression. }\end{array}$} & \multirow{3}{*}{$\begin{array}{l}\text { Hospitalization for } 24 \mathrm{~h} \text {, } \\
\text { confined to quarters for } \\
72 \mathrm{~h} \text { and not allowed to } \\
\text { return to flying until } \\
\text { cleared by flight surgeon. }\end{array}$} \\
\hline & USMC & & & & \\
\hline & \multirow[t]{3}{*}{ Air station Hospital } & & & & \\
\hline & & & & & $\begin{array}{l}\text { Consultation and } \\
\text { treatment with } \\
\text { physiatrist and PT. } \\
\text { PT referred to DC at } \\
4 \text { months. }\end{array}$ \\
\hline & & & & & $\begin{array}{l}\text { Pain free and return to } \\
\text { full function } 1 \text { month } \\
\text { after last chiropractic visit. }\end{array}$ \\
\hline \multirow{4}{*}{$\begin{array}{l}\text { Green, } 2008 \\
\text { [39] }\end{array}$} & United States & \multirow[t]{4}{*}{23 yo, male } & \multirow{4}{*}{$\begin{array}{l}\text { LBP (persistent } \\
\text { synchondrosis } \\
\text { of primary sacral } \\
\text { ossification center) }\end{array}$} & \multirow{4}{*}{$\begin{array}{l}\text { Treatment: HVLA } \\
\text { SMT of sacroiliac } \\
\text { joints, stretching, } \\
\text { conditioning } \\
\text { strengthening and } \\
\text { exercises, NSAIDs, } \\
\text { advice. Frequency: } \\
\text { initial treatment - }\end{array}$} & Referred to attending \\
\hline & Marine & & & & \\
\hline & \multirow{2}{*}{$\begin{array}{l}\text { Naval Medical } \\
\text { Center San } \\
\text { Diego }\end{array}$} & & & & $\begin{array}{l}\text { Insidious onset after } \\
\text { training exercise. }\end{array}$ \\
\hline & & & & & $\begin{array}{l}\text { At baseline: Verbal pain } \\
\text { scale } 7 / 10 \text { to } 9 / 10 \text { when }\end{array}$ \\
\hline
\end{tabular}


Table 1 Summary of location, population, condition of interest and key findings reported in included studies ( $n=20)$ (Continued)

\begin{tabular}{|c|c|c|c|c|c|}
\hline $\begin{array}{l}\text { First } \\
\text { Author, } \\
\text { Year } \\
\end{array}$ & $\begin{array}{l}\text { Country, Service } \\
\text { Branch, Service } \\
\text { Location, }\end{array}$ & $\begin{array}{l}\text { Population, } \\
\text { Number, } \\
\text { Groups }\end{array}$ & $\begin{array}{l}\text { Condition and } \\
\text { Duration }\end{array}$ & Treatment & $\begin{array}{l}\text { Key Findings: visit-specific } \\
\text { information, outcomes, } \\
\text { adverse events }\end{array}$ \\
\hline & & & & $\begin{array}{l}2 \text { weeks; } 6 \text { weeks after } \\
\text { consulting GMO further }\end{array}$ & $\begin{array}{l}\text { severe; RMDQ: 14/24; no } \\
\text { neuro deficits }\end{array}$ \\
\hline & & & & $\begin{array}{l}\text { Investigation; recommence } \\
\text { treatment - 4wks. }\end{array}$ & Discharged and full RTD. \\
\hline \multirow{5}{*}{$\begin{array}{l}\text { Green, } \\
2010 \text { [41] }\end{array}$} & United States & \multirow{5}{*}{$\begin{array}{l}\text { Instructor pilot } \\
38 \text { yo male }\end{array}$} & \multirow{5}{*}{$\begin{array}{l}\text { Uncomplicated } \\
\text { mechanical } \\
\text { neck pain }\end{array}$} & Treatment: 4 visits over & \multirow{2}{*}{$\begin{array}{l}\text { Intermittent neck pain } \\
\text { related to frequent flying } \\
\text { F/A-18 }\end{array}$} \\
\hline & \multirow{4}{*}{$\begin{array}{l}\text { Naval Medical } \\
\text { Center San Diego } \\
\text { Marine Corps }\end{array}$} & & & & \\
\hline & & & & $\begin{array}{l}\text { Included: active } \\
\text { stretching, HVLA } \\
\text { SMT, stretching and } \\
\text { strengthening home }\end{array}$ & $\begin{array}{l}\text { Referred for chiro care } \\
\text { after no change in } \\
\text { symptoms with } 2 \text { wks } \\
\text { acetaminophen }\end{array}$ \\
\hline & & & & ex & $\begin{array}{l}\text { At baseline: NRS 3/10; } \\
\text { NDI } 6 \% \text {; limited end } \\
\text { range of motion on } \\
\text { right; no neuro deficits }\end{array}$ \\
\hline & & & & & Resolved and full RTD \\
\hline \multirow{5}{*}{$\begin{array}{l}\text { Green, } \\
2014[40]\end{array}$} & United States & \multirow{5}{*}{$\begin{array}{l}\text { Helicopter } \\
\text { mechanic } \\
29 \text { yo, male }\end{array}$} & \multirow{5}{*}{$\begin{array}{l}\text { Mechanical } \\
\text { cervico-thoracic } \\
\text { pain \& myalgia }\end{array}$} & Interdisciplinary treatment & \multirow{2}{*}{$\begin{array}{l}\text { Chronic neck/upper } \\
\text { back pain of } 7 \text { yrs. post } \\
\text { flexion injury with } \\
\text { concurrent tinnitus, } \\
\text { dizziness and headaches }\end{array}$} \\
\hline & \multirow[t]{4}{*}{$\begin{array}{l}\text { Naval Medical } \\
\text { Center San } \\
\text { Diego Marine }\end{array}$} & & & $\begin{array}{l}\text { Chiropractic care: } 8 \text { visits; } \\
\text { HVLA SMT, soft tissue } \\
\text { mobilizations, advice, }\end{array}$ & \\
\hline & & & & $\begin{array}{l}\text { strengthening, proprioceptive); } \\
\text { Physical therapist care: } 5 \text { visits; } \\
\text { acupuncture }\end{array}$ & $\begin{array}{l}\text { Baseline: VPS } 7 / 10, \text { painful } \\
\text { limitation in motion, no } \\
\text { neuro deficits, x-rays-DDD, } \\
\text { right elongated styloid } \\
\text { process, left calcified } \\
\text { stylohyoid ligaments }\end{array}$ \\
\hline & & & & & $\begin{array}{l}\text { Treatment discontinued, } \\
\text { reported decrease } \\
\text { stiffness, VPS 4/10, no } \\
\text { adverse events }\end{array}$ \\
\hline & & & & & Returned to work. \\
\hline \multirow{3}{*}{$\begin{array}{l}\text { Lillie, } \\
2010 \text { [42] }\end{array}$} & United States & \multirow[t]{3}{*}{40 yo, male } & \multirow{3}{*}{$\begin{array}{l}\text { Acute episode } \\
\text { LBP with } \\
\text { radiculopathy }\end{array}$} & \multirow{3}{*}{$\begin{array}{l}\text { Interdisciplinary treatment, } \\
\text { with chiropractic care } \\
\text { provided over } 11 \text { visits in } \\
72 \text { days. Treatment included } \\
\text { HVLA and mechanically } \\
\text { assisted SMT, interferential } \\
\text { therapy, cryotherapy, moist } \\
\text { heat, nutritional and } \\
\text { psychosocial advice, } \\
\text { exercises. }\end{array}$} & \multirow{2}{*}{$\begin{array}{l}\text { Returned to regular } \\
\text { exercise routine and } \\
\text { able to perform all } \\
\text { required Navy } \\
\text { Physical Readiness } \\
\text { Tests. }\end{array}$} \\
\hline & \multirow[t]{2}{*}{$\begin{array}{l}\text { Navy Military } \\
\text { Treatment Facility } \\
\text { Chiropractic Clinic }\end{array}$} & & & & \\
\hline & & & & & $\begin{array}{l}\text { Subjective complaints } \\
\text { resolved and full RTD. }\end{array}$ \\
\hline \multirow{4}{*}{$\begin{array}{l}\text { Morgan, } \\
2014 \text { [43] }\end{array}$} & United States & \multirow{4}{*}{$\begin{array}{l}\text { Military officer } \\
25 \text { yo male }\end{array}$} & \multirow{4}{*}{$\begin{array}{l}\text { C3-5 ALL } \\
\text { heterotopic } \\
\text { ossification } \\
\text { and ankylosis }\end{array}$} & \multirow{4}{*}{$\begin{array}{l}\text { Interdisciplinary treatment } \\
\text { including oxycodone HCL/ } \\
\text { acetaminophen; chiropractic } \\
\text { care: } 1 / \text { wk. for } 13 \text { wks, then } \\
1 / \text { wk. for } 8 \text { wks, } 1 / 2 \text { wks for } \\
\text { 26wks - HVLA SMT thoracic } \\
\text { spine, respiratory therapy, } \\
\text { aqua therapy }\end{array}$} & \multirow{2}{*}{$\begin{array}{l}\text { Traumatic head injury } \\
\& \text { right femoral fracture } \\
\text { from motor vehicle } \\
\text { collision } 16 \text { months prior }\end{array}$} \\
\hline & \multirow[t]{3}{*}{$\begin{array}{l}\text { Walter Reed } \\
\text { National Military } \\
\text { Medical Center }\end{array}$} & & & & \\
\hline & & & & & $\begin{array}{l}\text { Baseline: neck and upper } \\
\text { back, bilateral hip, knee, } \\
\text { wrist, and shoulder pain; } \\
\text { VPS } 3 / 10 \text {; extremely } \\
\text { limited range neck } \\
\text { motion; restricted neck } \\
\text { \& thoracic joint motion; } \\
\text { decreased respiratory } \\
\text { excursion } 5 \mathrm{~cm} \text {; active } \\
\text { deep tendon reflexes; } \\
\text { increase CRP, ESR, } \\
\text { calcium, alkaline } \\
\text { phosphate }\end{array}$ \\
\hline & & & & & Normal chest expansion \\
\hline
\end{tabular}


Table 1 Summary of location, population, condition of interest and key findings reported in included studies $(n=20)$ (Continued)

\begin{tabular}{|c|c|c|c|c|c|}
\hline $\begin{array}{l}\text { Eirst } \\
\text { Author, } \\
\text { Year }\end{array}$ & $\begin{array}{l}\text { Country, Service } \\
\text { Branch, Service } \\
\text { Location, }\end{array}$ & $\begin{array}{l}\text { Population, } \\
\text { Number, } \\
\text { Groups }\end{array}$ & $\begin{array}{l}\text { Condition and } \\
\text { Duration }\end{array}$ & Treatment & $\begin{array}{l}\text { Key Findings: visit-specific } \\
\text { information, outcomes, } \\
\text { adverse events }\end{array}$ \\
\hline
\end{tabular}

increased to $3.5 \mathrm{~cm}$,

decrease pain

$\begin{array}{ll}\text { First } & \text { Country, Service } \\ \text { Author, } & \text { Branch, Service } \\ \text { Year } & \text { Location, } \\ \text { Dunn, } & \text { United States } \\ 2009 \text { [16] } & \text { DoD }\end{array}$

Population, $\quad$ Key Findings

Documents

2-option analysis System Related: chiropractic Legislative reports, care available at 49 designated policy documents, MTFs, planned expansion of published works 11 new locations in 2009-10; TRICARE chiropractic benefit available to active duty service members but not dependents.

Legislative History: chiropractic integrated in MHS as result of 10 pieces of legislation enacted over 17 yrs. (1993-2009).

Programmatic Growth: initiated as MHS demonstration project (1995); 5-fold increase in number of commands over $14 \mathrm{yr}$. period.

Leadership Structure: In MHS, leadership for chiropractic program at each command at department head or equivalent, usually two levels below hospital commanding officer. Each branch has Specialty Advisor responsible for issues related to chiropractic activities. No chiropractors functioning at DoD leadership levels. Decentralized structure of MHS and lack of chiropractor in leadership could impact integration.

Employment Status of Providers: Chiropractors in MHS serve in role of contractor or employee of contractors. Navy contracts directly with chiropractors (typically with no major benefits); Army and Air Force contracts with contracting organizations. Contractual relationships limited by contract period and if employees by contractors contract. Chiropractors in MHS may experience less job security and benefit "growth".

Clinical Work Duties: Chiropractors work within set of parameters (privileges) as established within system/facility, providing comprehensive chiropractic services (e.g. SMT, mobilizations, modalities, 
Table 1 Summary of location, population, condition of interest and key findings reported in included studies ( $n=20)$ (Continued)

\begin{tabular}{|c|c|c|c|c|c|}
\hline $\begin{array}{l}\text { Eirst } \\
\text { Author, } \\
\text { Year }\end{array}$ & $\begin{array}{l}\text { Country, Service } \\
\text { Branch, Service } \\
\text { Location, }\end{array}$ & $\begin{array}{l}\text { Population, } \\
\text { Number, } \\
\text { Groups }\end{array}$ & $\begin{array}{l}\text { Condition and } \\
\text { Duration }\end{array}$ & Treatment & $\begin{array}{l}\text { Key Findings: visit-specific } \\
\text { information, outcomes, } \\
\text { adverse events }\end{array}$ \\
\hline
\end{tabular}

rehabilitation), uphold
guidelines, and may perform
administrative tasks. Typically
supervised by non-chiropractor
officers. Quality assurance via
peer review. Informally,
chiropractors interact with
other providers in highly
transparent environment,
attend regular staff meetings,
provide in-service training,
maintain competencies, and
adhere to documentation
requirements.
Patient Access: Chiropractic
care accessed largely by
gatekeeper referral, which
may act as limiting factor.
Patients must be seen
within 30 days.
Patient Demographics: In
DoD, chiropractors care for
mix of active duty and active
duty veteran patients, most
likely for musculoskeletal
conditions.

Academic Affiliations and Research: First training rotation within DoD in 2001 with New York Chiropractic College at National Naval Medical Center. Two others established but closed. Little research conducted in DoD and no research time provided in contracts.

Mior, Canada

2018 [44] Canadian Armed Forces
Canadian Forces Health Services Key informant interviews: MD $(n=7), \mathrm{PT}$

$(n=13), \mathrm{DC}$ $(n=5)$
Participant perspectives to Barriers, Opportunities and Recommendations to Integrated Chiropractic Services within CFHS: Barriers:

1: Referring to Off-base Chiropractic Services (baseto-base Variation; Gatekeeper Roles; Care Delivery Unit Medical Officer or Lead physiotherapist designated to chiropractor referral role; Decision to refer to chiropractor based on individual clinician preference and experience, rather than a systematic approach).

2: Inter-professional Communication (Communication processes affected by site-specific resources and current practices; Current practices reflect clinician perspective and past experience; Written communication (referral, reports) not standardized; No dialogue between health care providers on base and chiropractors). 
Table 1 Summary of location, population, condition of interest and key findings reported in included studies ( $n=20)$ (Continued)

\begin{tabular}{|c|c|c|c|c|c|}
\hline $\begin{array}{l}\text { First } \\
\text { Author, } \\
\text { Year }\end{array}$ & $\begin{array}{l}\text { Country, Service } \\
\text { Branch, Service } \\
\text { Location, }\end{array}$ & $\begin{array}{l}\text { Population, } \\
\text { Number, } \\
\text { Groups }\end{array}$ & $\begin{array}{l}\text { Condition and } \\
\text { Duration }\end{array}$ & Treatment & $\begin{array}{l}\text { Key Findings: visit-specific } \\
\text { information, outcomes, } \\
\text { adverse events }\end{array}$ \\
\hline
\end{tabular}

\author{
3. Duplication of Health Care \\ Services (Scope of practice \\ change: physical therapists \\ and chiropractors; Difficulty \\ distinguishing chiropractor as \\ a profession rather than an \\ intervention; Non-uniform \\ personnel, e.g. chiropractor \\ not able to deploy) Opportunities: \\ 1. Musculoskeletal Disorders \\ (Prevalence of MSK conditions, \\ provide care which is clinical \\ and cost effective) \\ 2: Inter-professional Collaborative \\ Care (Collaborative, integrated, \\ patient-centered care; Base-to- \\ base variation dependent upon \\ location, size, resources and primary \\ purpose; Co-location of providers \\ strengthens inter-professional \\ communication and relationships) \\ 3: Evidence-Based Approach \\ (Standardization of clinical care \\ using clinical practice guidelines \\ based upon high quality evidence) \\ 4: The Spectrum of Care (Knowledge \\ of CAF spectrum of care; Utilize \\ chiropractors' full scope of practice) \\ Recommendations: \\ 1. First establish personal rather \\ than professional-level relationships \\ 2. Explicate role and responsibilities \\ of chiropractor based on scope of \\ practice \\ 3. Standardize communication and \\ treatment plans respectful of military \\ culture
}

\begin{abstract}
Acronyms: $A D L$ activities of daily living, $A L L$ anterior longitudinal ligament, BPFS back pain functional scale, CAF Canadian Armed Forces, $C A M$ complementary and alternative medicine, CFB Canadian Forces Base, CFHS Canadian Forces Health Services, Cl confidence interval, CMT chiropractic manipulative therapy, CRP C reactive protein, $D C$ chiropractor, $D D D$ degenerative disc disease, $D o D$ Department of Defense, ESR erythrocyte sedimentation rate, $F / A$ fighter/attack, $f / u$ followup, GMO general medical officer, HR hazards ratio, HVLA SMT high velocity low amplitude spinal manipulative therapy, $L B P$ low back pain, $M D$ medical doctor, mobs mobilization, MHS military health system, MSK musculoskeletal, MTF military treatment facility, NDI neck disability index, NHIS National Health Interview Survey, NRS numerical pain rating scale, OR odds ratio, PT physical therapist, RAAF Royal Australian Air Force, RMDQ Roland-Morris Disability Questionnaire, RTD return to duty, SD standard deviation, SE standard error, SMC standard medical care, SOF special operation forces, TBI traumatic brain injury, VPS verbal pain scale, UMC usual medical care, USMC United States Marine Corps, wks weeks, yo years old, yr. years
\end{abstract}

\section{Chiropractic scope of practice}

The practice of chiropractic is the assessment of conditions related to the spine, nervous system, and joints and the diagnosis, prevention, and treatment of these conditions [46]. However, in Military Treatment Facilities, chiropractic scope of practice is established by the system/facility and may include comprehensive services (e.g. spinal manipulative therapy (SMT), mobilizations, modalities, rehabilitation), utilization of guidelines, and in some instances the performance of administrative tasks [16].
The chiropractic scope of practice described in the included articles consisted of both assessment and treatment. The chiropractic assessment included a focused history, physical examination, clinical impressions, disability, prognosis, and treatment plan elements. The interventions described in the articles included the following physical techniques and modalities: joint manipulation and mobilization of the spine and extremities [12, 29, 30, 38-42], soft tissue massage [12, 29], stretching/proprioceptive neurological facilitation maneuvers [12, 38-41], cryotherapy [30, 42], moist heat [42], superficial heat [30], 
McKenzie exercises [29], exercise [12, 29, 30, 38-42], interferential current [12, 30, 42], ultrasound [30], acupuncture [12], myofascial release [38], ischemic compression [38], advice on activities of daily living [29], postural/ ergonomic advice [29, 39, 40], as well as nutritional and psychosocial aspects of treatment [42].

\section{Delivery model and benefits}

In the US, active duty military personnel predominantly attended an on-base clinic [16, 29, 30, 38-43]. Care was described as interdisciplinary; however, healthcare providers typically provided services in physical isolation from other care providers. Case discussions occurred most often when prompted by a referral. In this healthcare model, the service member's first point of contact is with a primary care provider who refers to chiropractic care if deemed necessary.

Chiropractors in the Military Health System are either contractors or employees of contractors, typically without healthcare and other benefits as part of their employment contract [16]. The chiropractor's employment period is limited to the contract period. A decentralized leadership Military Health System structure may challenge the integration of chiropractic service into the Military Health System. Chiropractic care is a TRICARE benefit available to active duty service personnel but not their dependents [16].

\section{Challenges to collaboration}

Challenges to collaboration were identified in two qualitative studies. Mior et al. described barriers to the integration of chiropractic services within the Canadian Forces Health Services (CFHS) [44]. Challenges to the integration of chiropractic services within the CFHS included base-tobase variation in referral procedures, which were associated with clinician preference and experience rather than a systematic approach. Mior et al. also reported that interprofessional communication varied by base and typically lacked standardized reporting [44]. The minimal reported interaction between chiropractors and CFHS healthcare providers apparently impeded the development of a positive inter-professional relationship. Chiropractic care was perceived as a duplication of physical therapy services, often considered more as a single intervention, that is spinal manipulative therapy, rather than as a profession.

Dunn et al. [16] identified that untimely access, unavailable services, and unobtained referrals could affect the integration of chiropractic services in the Military Health System. Despite the legislative mandates in the US, Dunn et al. argued that continued acceptance and integration will depend upon the chiropractors adding measurable value to service delivery [16].

Both qualitative reports suggested that improved collaboration and/or integration was not possible without service availability at military treatment facilities. Also, ensuring care provided was consistent with the needs of the patients, while being respectful of the roles and responsibilities of others, was argued as important to ensure sustainable integration $[16,44]$.

\section{Opportunities}

Opportunities to include chiropractic services within the active duty military were identified in several articles $[16$, 31, 44]. These opportunities included providing clinical, cost effective evidence-based care for musculoskeletal conditions within an environment of inter-professional collaborative care. Specifically, within this environment the delivery of care would be based on clinical practice guidelines that draw upon the full scope of chiropractors' practice rather than solely the delivery of a specific intervention.

\section{Utilization of chiropractic services}

The utilization rate of chiropractic services by active duty military members was reported in seven cross-sectional surveys $[28,32-37]$. The reported utilization rate of chiropractic services over the preceding 12 months was consistent over time (2000-2011) in the United States but then decreased in 2013. Specifically, the utilization rate ranged between 5.2 and $10.5 \%$ among active duty military personnel $[28,32,35-37]$ and $14.8 \%$ in the Reserve/National Guard in 2007 [35], to a low of $2.9 \%$ in 2013 among services in the Military Health System [49]. In Australian military aircrew, $12 \%$ sought chiropractic services for flight-related neck pain [33], which is higher than the 12-month chiropractic service utilization in the US in the period 2008-2011.

\section{Type of conditions treated and duration of treatment period}

Back pain with or without radiculopathy accounted for the majority of presentations [12, 29, 30, 38, 39]. In a US cross-sectional study, $42.7 \%$ of respondents reported using chiropractic services for low back pain (LBP), $27.3 \%$ for headaches, and $10.9 \%$ for general health, wellness, and prevention [31]. In a Canadian cross-sectional survey, $97 \%$ of patients reported spine-related musculoskeletal complaints, of which $52 \%$ were LBP, and $3 \%$ involved conditions of the extremities [12].

Neck pain was reported as the reason for chiropractic consult in several articles [12, 33, 40, 41, 43]. In a survey of Australian air force personnel, $12 \%$ of the respondents sought chiropractic care for neck pain [33]. Neck pain with radiating symptoms was reported in one crosssectional study [12]. Aside from one of the included randomized controlled trials [29], most articles reported patients presenting with chronic musculoskeletal conditions [12, 30, 31, 40, 43].

Chiropractic treatment duration was reported in nine articles and varied considerably between articles [12, 29, 
30, 38-43]. In their cross-sectional study, Boudreau et al. [12] reported the average number of chiropractic visits as $5.7 \pm 4.1$ (mean $\pm \mathrm{SD}$ ), ranging from one to 25 visits. Goertz et al. [29] in their randomized control trial (RCT) scheduled up to two chiropractic visits weekly (eight visits) for a period of 4 weeks, and reported participants attended an average of seven visits. In another randomized controlled trial, patients were allocated to up to 12 visits over 6 weeks [30], with patients utilizing a much smaller number than the available number of visits. Patients who attended at least one visit to a chiropractor reported a mean number of chiropractic visits (mean $\pm \mathrm{SD}$ ) which varied across study sites and ranged from 2.3 \pm 1.4 (San Diego), $4.7 \pm 2.5$ (Walter Reed), to $5.4 \pm 2.6$ (Pensacola) [30]. In a survey of US Military Treatment Facilities offering chiropractic services, the average number of patient visits to a chiropractor was 5.4, the highest reported patient visits of any complementary and alternative medicine provider in 2013 [31].

Case report participants reported the greatest number of chiropractic visits. The number varied from 16 visits over 30 weeks with an aviator instructor with acute LBP [38], a military officer with C3-5 anterior longitudinal ligament heterotopic ossification and ankyloses received 34 visits over 47 weeks [43], and a Naval Petty Officer with low back and radicular pain attended 11 visits over 72 days [42].

\section{Outcomes of care}

Reported outcomes of chiropractic care were predominantly positive. Favourable outcomes following chiropractic care were reported in each of the case reports, however in one case report the patient improved but did not return to duty [43]. In a Canadian cross-sectional study, active duty military reported satisfaction with care $(94.2 \%)$ [12]. Further, all physicians in this study identified a perceived demand for chiropractic services, and the majority (80.6\%) were satisfied with chiropractic services [12].

In a RCT, Goertz et al. [29] reported a mean difference favouring chiropractic manipulative therapy (CMT) in addition to standard medical care (SMC) over SMC alone for each of the primary outcomes at 2 and 4 weeks in acute LBP subjects. A greater percentage of participants in the SMC plus CMT group (73\%) rated their global improvement as pain completely gone, much better, or moderately better, compared with $17 \%$ in the SMC group. Similarly in another RCT, Goertz et al. [30] reported mean differences favouring usual medical care (UMC) with chiropractic care (CC) over UMC alone for each of the primary outcomes at 6 and 12 weeks, although the magnitude of difference decreased at 12 weeks. Additionally, secondary outcomes of worst LBP intensity and symptom bothersomeness also favoured UMC + CC over UMC. Overall, $\mathrm{UMC}+\mathrm{CC}$ identified better global perceived improvement, satisfaction with care, and used less pain medication.

The DeVocht et al. [45] RCT assessed if a short course (4 visits) of CMT improved reaction and response time outcomes in special operation forces military compared to wait-list control. Despite observing an immediate effect after the first session on complex response task, no significant between group differences were reported for any of the outcome measures at 2 weeks.

\section{Adverse events}

Adverse events were reported in three articles, all RCTs $[29,30,45]$. Although there were no serious adverse events reported, two studies reported 6 minor events of which 5 were unrelated to trial procedures and 1 related to SMT [45]. In a large RCT [33], there were 62 events reported, where 19 were in the usual care group and 43 in the usual care and chiropractic care group. The majority (49/62) were reported as muscle or joint stiffness related to either chiropractic care, physiotherapy care or self-care.

\section{Discussion}

We found 20 articles that described chiropractic services within the active military worldwide. The majority of articles $(n=17)$ were from the US, with additional information provided from articles from Canada $(n=2)$ and Australia $(n=1)$. The majority were cross-sectional studies assessing utilization or access of chiropractic services or case reports highlighting common or unique conditions managed; there were only three RCTs evaluating chiropractic as an intervention in this setting. The majority of included articles were published between 2006 and $2010(n=9)$ but the more robust designed clinical studies were published in the last 2 years. There is less research related to chiropractic services in active military personnel compared to that involving veterans $[16,47]$.

We found that chiropractic services are provided to active duty military in on-base clinical facilities in only one country, the US; this is driven by US legislation [16]. Regardless of location, services are typically accessed through a gatekeeper, usually a medical physician [16, 44]. Care delivery models vary but the extent of integration of chiropractic services within the US Military Health System remains unclear. In the US and Canada, chiropractic services are available to active duty military; however, they are delivered by paid contracted chiropractors in the US, as opposed to a third party insured military personnel benefit in Canada. It is unclear what service delivery models exist in countries other than the US and Canada as we located no articles describing this. However, it appears that the inherent gatekeeper referral processes influence the access to chiropractic services. 
Reported 12-month utilization of chiropractic services in the US ranged from 2.9 to $10.5 \%$ between 2000 and 2013 [28, 32, 36, 37]. Outside of the US, we found only one study reporting a $12 \%$ utilization of chiropractic services among Australian military aircrew [33]. The reported utilization seems similar to that reported in the general population [48].

Aside from one randomized controlled trial which included acute LBP patients, most articles reported patients presenting with chronic musculoskeletal conditions. These findings are similar to those seen in the general population, where musculoskeletal conditions are the predominant reason for consulting chiropractors [48]. The frequency of chiropractic visits reported in cross-sectional studies ranged from a mean of 5.4 in a survey of US Military Treatment Facilities [31] to 5.7 in a single Canadian base [12]. In Canada, limits to covered benefits and policy may influence visit frequency.

Outcomes of care provided by chiropractors was positive in most of the reported clinical studies; however, six of these were case report designs that cannot evaluate effectiveness. In two included RCTs, outcomes favoured usual medical care and chiropractic care compared to standard medical care alone. These findings are consistent with recent LBP guidelines supporting the use of manual and conservative care [49-51]. However, in the larger LBP pragmatic trial [30], treatment included interventions of questionable effectiveness [51]; suggesting further clinical trial data are required to assess if practice is consistent with current guidelines.

In another RCT, the use of a short course (4 visits over 2 weeks) of chiropractic manual therapy to improve select measures of performance in special operations military personnel was no different from wait-list control [45]. Despite empirical evidence of performance enhancement following manual therapy, the immediate but not statistically significant longer-term effect reported in this study is consistent with findings in systematic reviews assessing the impact of manual therapy on performance [52, 53].

Our review adds to a previous review examining the integration of chiropractic services in military and veteran health care facilities [47]. We add new information assessing chiropractic services in active duty military from articles in the US, Canada, and Australia. Unfortunately, we found no evidence of chiropractic services provided to active duty military in other countries. The majority of the articles emanated from the US where chiropractic services were included in the Military Health System since 1995 [47]. Little is known about the nature of integration of chiropractic services in the US Military Health System, but evidence suggests that it varies from base-to-base [16, 47]. We add new information from Canada highlighting the challenges and opportunities of the inclusion of chiropractic services in active duty military [44]. Given utilization data is limited to the US and Australian Air Force, further descriptive studies are required to fill this gap worldwide.

Understanding the characteristics of chiropractic services provided is important in assessing and maximizing quality of care [54]. The significant expansion of chiropractic services within the US Military Health System has been largely driven by legislative directives, which in themselves may challenge the nature and extent of system integration [16]. If value of services is measured by system needs rather than that of the providers [16], then system and care-based outcomes are important assessment metrics required to ensure continued success. Our scoping review suggests little is known about the clinical and quality metrics of chiropractic services in active duty military globally. Qualitative studies could provide the necessary understanding of the system and resource barriers and potential opportunities for inclusion or expansion of chiropractic services worldwide.

\section{Strengths and limitations}

A strength of our scoping review was the systematic process used to collect and summarize the evidence from this diverse body of literature. A scoping review is the most appropriate method to collect and organize diverse information and to develop a picture of the existing evidence base when a broad research question is asked [55]. Our health sciences librarian conducted a broad and methodologically rigorous literature search, which was reviewed by a second librarian. Further, we searched two military specific databases in an effort to capture all discipline specific relevant articles. Study selection was based upon detailed inclusion and exclusion criteria to ensure that consensus between paired independent reviewers was transparent and reproducible.

There are limitations in this review. In keeping with the scoping review framework we collated the evidence on chiropractic care in the military and did not critically appraise the methodology of the reported articles [19]. Future systematic reviews focusing on the specific factors discussed in this review should include an appraisal of the study methods. We restricted our search to include articles in the English language, which may have excluded some relevant articles. However, chiropractic journals publish in English, which is recognized as the standard language of science, thereby reducing this risk [56].

\section{Conclusion}

Our scoping review explored the available evidence related to chiropractic services within active duty military. The majority of the articles emanated from the US and were cross-sectional in nature. Two recent RCTs provide evidence of comparative effectiveness of adding chiropractic 
care to usual medical care. Despite the reported use of chiropractic services in Australia, Canada, and the US, there is little available published evidence related to the nature, use, and outcomes of chiropractic care in active duty military. Our review suggests the need for further qualitative, descriptive, and clinical trial data worldwide to inform the role and value of chiropractic services in active duty military globally.

\section{Additional files}

Additional file 1: Search Strategy (DOCX $21 \mathrm{~kb}$ )

\section{Acknowledgements}

The authors would like to acknowledge the invaluable contributions to this scoping review by Kent Murnaghan.

\section{Authors' contributions}

Made substantial contributions to conception and design, or acquisition of data, or analysis and interpretation of data;-DS, SM, DT, CC, ATV. Been involved in drafting the manuscript or revising it critically for important intellectual contentDS, SM, DT, CC, SF, ATV, PC. Each author should have participated sufficiently in the work to take public responsibility for appropriate portions of the content; and-DS, SM, DT, CC, SF, ATV, PC. Agreed to be accountable for all aspects of the work in ensuring that questions related to the accuracy or integrity of any part of the work are appropriately investigated and resolved-DS, SM, DT, CC, SF, ATV, PC. All authors read and approved the final manuscript.

\section{Funding}

This research was undertaken, in part, thanks to funding from the Canadian Chiropractic Association and the Ontario Chiropractic Association to the Centre for Disability Prevention and Rehabilitation at the University of Ontario Institute of Technology. The funding sources had no role in the design, execution, analyses or interpretation of the data, or decision to submit results.

\section{Availability of data and materials}

Not applicable.

\section{Ethics approval and consent to participate}

This research did not involve human subjects, human material or human data. Ethics approval and consent are not applicable.

\section{Consent for publication}

Not applicable. Manuscript does not include details, images or videos relating to an individual person.

\section{Competing interests}

DS, SM, CC, PC and SF have received research funding from the Canadian national and provincial chiropractic organizations, either as salary support or for research project funding. SF is Deputy-Editor-in-Chief for Chiropractic and Manual Therapies; however, he did not have any involvement in the editorial process for this manuscript and was blinded from the editorial system for this paper from submission to decision.

\section{Author details}

${ }^{1}$ UOIT-CMCC Centre for Disability Prevention and Rehabilitation, University of Ontario Institute of Technology (UOIT) and Canadian Memorial Chiropractic College (CMCC), 6100 Leslie Street, Toronto, Ontario M2H 3J1, Canada. ${ }^{2}$ Department of Research and Innovation, Canadian Memorial Chiropractic College, 6100 Leslie Street, Toronto, Ontario M2H 3J1, Canada. ${ }^{3}$ Department of Graduate Studies, Canadian Memorial Chiropractic College, 6100 Leslie Street, Toronto, Ontario M2H 3J1, Canada. ${ }^{4}$ Faculty of Health Sciences, University of Ontario Institute of Technology (UOIT), 2000 Simcoe Street North, Oshawa, Ontario L1G 0C5, Canada. 5 Department of Chiropractic, Faculty of Science and Engineering, Macquarie University, Level 3, 17 Wally's Walk, North Ryde, NSW 2109, Australia. ${ }^{6}$ Faculty of Health Sciences, University of Ontario Institute of Technology (UOIT), 2000 Simcoe Street North, Oshawa, Ontario L1G 0C5, Canada.

Received: 20 February 2019 Accepted: 22 May 2019

Published online: 15 July 2019

\section{References}

1. Spooner SP, Tyner SD, Sowers C, Tsao J, Stuessi K. Utility of a sports medicine model in military combat concussion and musculoskeleta restoration care. Mil Med. 2014;179:1319-24.

2. Rowe $\mathrm{P}$, Hébert LJ. The impact of musculoskeletal conditions on the Canadian forces. In: Aiken A, Bélanger S, editors. Shaping the future: military and veteran health research. Kingston: Canadian Defence: Academy Press; 2011. p. 377-91

3. Litow CD, Krahl PL. Public health potential of a disability tracking system: analysis of U.S. navy and marine corps physical evaluation boards 20052006. Mil Med. 2007;172:1270-4.

4. Born J, Hawes R, Whitehead J. Results from health and lifestyle information survey of Canadian forces personnel 2008/2009. In: Regular Force version: your health speaks volumes. Ottawa: Government of Canada; 2010.

5. Canadian Forces Health Services Group. Surgeon General's Report 2014: Consolidation, Innovation, Readiness. Ottawa: Government of Canada, Department of National Defence; 2014.

6. Ang B, Harms-Ringdahl K. Neck pain and related disability in helicopter pilots: a survey of prevalence and risk factors. Aviat Space Environ Med. 2006:77:713-9.

7. Wickes S, Scott J, Greeves J. Epidemiology of flight-related neck pain in Royal air Force (RAF) aircrew. Aviation, space, and environmental medicine, vol. 76; 2006. p. 298

8. Hiebert R, Campello MA, Weiser S, Ziemke GW, Fox BA, Nordin M. Predictors of short-term work-related disability among active duty US navy personnel: a cohort study in patients with acute and subacute low back pain. Spine J. 2012:12:806-16

9. Smith L, Westrick R, Sauers S, et al. Underreporting of musculoskeletal injuries in the US army: findings from an infantry brigade combat survey. Sports Health 2016; 8(6):507-513

10. Canada Parliament House of Commons. Caring for Canada's III and Injured Military Personnel (June 2014) $41^{\text {st }}$ Parliament, $2^{\text {nd }}$ Session. In: Standing Committee on National Defence. Ottawa: Government of Canada; 2014. p. 1-93.

11. Cote P, Wong JJ, Sutton D, et al. Management of neck pain and associated disorders: a clinical practice guideline from the Ontario protocol for traffic injury management (OPTIMa) collaboration. Eur Spine J. 2016;25:2000-22.

12. Boudreau LA, Busse JW, McBride G. Chiropractic services in the Canadian Armed Forces: a pilot project. Mil Med. 2006;171:572-6.

13. McGee JC, Bise CG, Stevens JM. An orthopedic performance improvement initiative at a small military treatment facility. Mil Med. 2017;182:18-25.

14. Brawley S, Fairbanks K, Nguyen W, Blivin S, Frantz E. Sports medicine training room clinic model for the military. Mil Med. 2012;177:135-8.

15. Kelly EW, Bradway LF. A team approach to the treatment of musculoskeletal injuries suffered by navy recruits: a method to decrease attrition and improve quality of care. Mil Med. 1997;162:354-9.

16. Dunn AS, Green BN, Gilford S. An analysis of the integration of chiropractic services within the United States military and veterans' health care systems. J Manip Physiol Ther. 2009:32:749-57.

17. Green BN, Johnson CD, Lisi AJ, Tucker J. Chiropractic practice in military and veterans health care: the state of the literature. J Can Chiropr Assoc. 2009: 53:194-204.

18. Lisi AJ, Brandt CA. Trends in the use and characteristics of chiropractic services in the Department of Veterans Affairs. J Manip Physiol Ther. 2016; 39:381-6.

19. Arksey H, O'Malley L. Scoping studies: towards a methodological framework Int J Soc Res Methodol. 2005;8:19-32.

20. Levac D, Coquhoun H, O'Brien K. Scoping studies: advancing the methodology. Implement Sci. 2010;5:1-9.

21. O'Brien KK, Colquhoun $\mathrm{H}$, Levac D, et al. Advancing scoping study methodology: a web-based survey and consultation of perceptions on terminology, definition and methodological steps. BMC Health Serv Res. 2016;16:305

22. Tricco AC, Lillie $E$, Zarin W, et al. PRISMA extension for scoping reviews (PRISMA-SCR): checklist and explanation. Ann Intern Med. 2018;169:467-73. 
23. McGowan J, Sampson M, Salzwedel DM, Cogo E, Foerster V, Lefebvre C. PRESS peer review of electronic search strategies: 2015 guideline statement. J Clin Epidemiol. 2016;75:40-6.

24. Sampson M, McGowan J, Cogo E, Grimshaw J, Moher D, Lefebvre C. An evidence-based practice guideline for the peer review of electronic search strategies. J Clin Epidemiol. 2009:62:944-52.

25. US National Library of Medicine. MeSH.

26. Thomas Reuters. ENDNOTE.

27. Pawson R. Evidence-based policy: the promise of 'realist synthesis'. Evaluation. 2002:8:340-58.

28. Goertz C, Marriott BP, Finch MD, et al. Military report more complementary and alternative medicine use than civilians. J Altern Complement Med. 2013;19:509-17.

29. Goertz CM, Long CR, Hondras MA, et al. Adding chiropractic manipulative therapy to standard medical care for patients with acute low back pain: results of a pragmatic randomized comparative effectiveness study. Spine. 2013;38:627-34.

30. Goertz CM, Long CR, Vining RD, Pohlman KA, Walter J, Coulter I. Effect of usual medical care plus chiropractic care vs usual medical care alone on pain and disability among US service members with low back pain: a comparative effectiveness clinical trial. JAMA Netw Open. 2018;1:1-15.

31. Herman PM, Sorbero ME, Sims-Columbia AC. Complementary and alternative medicine services in the Military Health System. J Altern Complement Med 2017:23:837-43

32. Jacobson IG, White MR, Smith TC, et al. Self-reported health symptoms and conditions among complementary and alternative medicine users in a large military cohort. Ann Epidemiol. 2009;19:613-22.

33. Netto K, Hampson G, Oppermann B, Carstairs G, Aisbett B. Management of neck pain in Royal Australian air Force fast jet aircrew. Mil Med. 2011;176:106-9.

34. Petri RP Jr, Delgado RE. Integrative medicine experience in the U.S. Department of Defense. Med Acupunct. 2015;27:328-34.

35. Ryan MA, Smith TC, Smith B, et al. Millennium cohort: enrollment begins a 21-year contribution to understanding the impact of military service. J Clin Epidemiol. 2007; 60:181-91.

36. Smith TC, Smith B, Ryan MA. Prospective investigation of complementary and alternative medicine use and subsequent hospitalizations. BMC Complement Altern Med. 2008;8:19.

37. White MR, Jacobson IG, Smith B, et al. Health care utilization among complementary and alternative medicine users in a large military cohort. BMC Complement Altern Med. 2011;11:27.

38. Green BN, Sims J, Allen R. Use of conventional and alternative treatment strategies for a case of low back pain in a F/A-18 aviator. Chiropr Osteopat. 2006;14:11.

39. Green BN, Schultz G, Stanley M. Persistent synchondrosis of a primary sacral ossification center in an adult with low back pain. Spine J. 2008:8:1037-41.

40. Green BN, Browske LK, Rosenthal CM. Elongated styloid processes and calcified stylohyoid ligaments in a patient with neck pain: implications for manual therapy practice. J Chiropr Med. 2014;13:128-33.

41. Green BN, Dunn AS, Pearce SM, Johnson CD. Conservative management of uncomplicated mechanical neck pain in a military aviator. J Can Chiropr Assoc. 2010;54:92-9.

42. Lillie GR. Resolution of low back and radicular pain in a 40-year-old male United States navy petty officer after collaborative medical and chiropractic care. J Chiro Med. 2010;9:17-21.

43. Morgan WE, Morgan CP. Chiropractic care of a patient with neurogenic heterotopic ossification of the anterior longitudinal ligament after traumatic brain injury: a case report. J Chiropr Med. 2014;13:260-5.

44. Mior SA, Vogel E, Sutton D, et al. Exploring chiropractic services in the Canadian forces health services - perceptions of facilitators and barriers among key informants. Mil Med 2019; 184(5-6):e344-e351.

45. DeVocht JW, Vining R, Smith DL, Long C, Jones T, Goertz C. Effect of chiropractic manipulative therapy on reaction time in special operations forces military personnel: a randomized controlled trial. Trials. 2019:20:5.

46. College of Chiropractors of Ontario. Scope of Practice and Authorized Acts. 2018.

47. Green BN, Johnson CD, Daniels CJ, Napuli JG, Gliedt JA, Paris DJ. Integration of chiropractic Services in Military and Veteran Health Care Facilities: a systematic review of the literature. J Evid Based Complementary Altern Med. 2016:21:115-30.

48. Beliveau PJH, Wong JJ, Sutton DA, et al. The chiropractic profession: a scoping review of utilization rates, reasons for seeking care, patient profiles, and care provided. Chiropr Man Therap. 2017;25:35.
49. Chou R, Cote P. The global spine care initiative: applying evidence-based guidelines on the non-invasive management of back and neck pain to lowand middle-income communities, vol. 27; 2018. p. 851-60.

50. Foster NE, Anema JR, Cherkin $\mathrm{D}$, et al. Prevention and treatment of low back pain: evidence, challenges, and promising directions. Lancet. 2018;391:2368-83.

51. Wong JJ, Cote P, Sutton DA, et al. Clinical practice guidelines for the noninvasive management of low back pain: a systematic review by the Ontario protocol for traffic injury management (OPTIMa) collaboration. Eur J Pain. 2017:21:201-16.

52. Botelho MB, Alvarenga BAP, Molina N, Ribas M, Baptista AF. Spinal manipulative therapy and sports performance enhancement: a systematic review. J Manip Physiol Ther. 2017;40:535-43.

53. Miners AL. Chiropractic treatment and the enhancement of sport performance: a narrative literature review. J Can Chiropr Assoc. 2010;54:210-21.

54. Lisi AJ, Khorsan R, Smith MM, Mittman BS. Variations in the implementation and characteristics of chiropractic services in VA. Med Care. 2014;52:597-104.

55. Armstrong R, Hall BJ, Doyle J, Waters E, Update C. Scoping the scope' of a cochrane review. J Public Health (Oxf). 2011;33:147-50.

56. Green BN. Improvements in the Journal of Chiropractic Education for 2013. J Chiropr Educ. 2013:27:1-4.

\section{Publisher's Note}

Springer Nature remains neutral with regard to jurisdictional claims in published maps and institutional affiliations.
Ready to submit your research? Choose BMC and benefit from:

- fast, convenient online submission

- thorough peer review by experienced researchers in your field

- rapid publication on acceptance

- support for research data, including large and complex data types

- gold Open Access which fosters wider collaboration and increased citations

- maximum visibility for your research: over $100 \mathrm{M}$ website views per year

At BMC, research is always in progress.

Learn more biomedcentral.com/submissions 